\title{
Macular Pigment, Visual Function, and Macular Disease among Subjects with Alzheimer's Disease: An Exploratory Study
}

\author{
John M. Nolan ${ }^{\mathrm{a}, *}$, Ekaterina Loskutova ${ }^{\mathrm{a}}$, Alan N. Howard ${ }^{\mathrm{b}, \mathrm{c}}$, Rachel Moran ${ }^{\mathrm{a}}$, Riona Mulcahy ${ }^{\mathrm{d}}$, \\ Jim Stack ${ }^{\mathrm{a}}$, Maggie Bolger ${ }^{\mathrm{d}}$, Jessica Dennison ${ }^{\mathrm{a}}$, Kwadwo Owusu Akuffo ${ }^{\mathrm{a}}$, Niamh Owens ${ }^{\mathrm{a}}$, \\ David I. Thurnham ${ }^{\mathrm{e}}$ and Stephen Beatty ${ }^{\mathrm{a}}$ \\ ${ }^{a}$ Macular Pigment Research Group, Department of Chemical and Life Sciences, Waterford Institute of Technology, \\ Waterford, Ireland \\ ${ }^{\mathrm{b}}$ Howard Foundation, Cambridge, UK \\ ${ }^{\mathrm{c}}$ Downing College, University of Cambridge, Cambridge, UK \\ ${ }^{\mathrm{d}}$ Waterford Regional Hospital, Age-Related Care Unit, Waterford, Ireland \\ ${ }^{\mathrm{e}}$ Northern Ireland, Centre for Food and Health (NICHE), University of Ulster, Coleraine, UK
}

Accepted 2 May 2014

\begin{abstract}
.
Background: The macula (central retina) contains a yellow pigment, comprising the dietary carotenoids lutein (L), zeaxanthin (Z), and meso-zeaxanthin, known as macular pigment (MP). The concentrations of MP's constituent carotenoids in retina and brain tissue correlate, and there is a biologically-plausible rationale, supported by emerging evidence, that MP's constituent carotenoids are also important for cognitive function.

Objective: To investigate if patients with Alzheimer's disease (AD) are comparable to controls in terms of MP and visual function.

Methods: 36 patients with moderate AD and 33 controls with the same age range participated. MP was measured using dual-wavelength autofluorescence (Heidelberg Spectralis ${ }^{\circledR}$ ); cognitive function was assessed using a battery of cognition tests (including Cambridge Neuropsychological Test Automated Battery). Visual function was recorded by measuring best corrected visual acuity (BCVA) and contrast sensitivity (CS). Serum L and Z concentrations (by HPLC) and age-related macular degeneration (AMD, by retinal examination) status were also assessed.

Results: In the AD group, central MP (i.e., at $\left.0.23^{\circ}\right)$ and MP volume were significantly lower than the control group ( $p<0.001$ for both), as were measures of BCVA, CS, and serum $\mathrm{L}$ and $\mathrm{Z}$ concentrations ( $p<0.05$, for all).

Conclusion: AD patients were observed to exhibit significantly less MP, lower serum concentrations of $\mathrm{L}$ and $\mathrm{Z}$, poorer vision, and a higher occurrence of AMD when compared to control subjects. A clinical trial in AD patients designed to investigate the impact of macular carotenoid supplementation with respect to MP, visual function, and cognitive function is merited.
\end{abstract}

Keywords: Age-related macular degeneration, Alzheimer's disease, cognitive function, contrast sensitivity, lutein, meso-zeaxanthin, visual function, zeaxanthin

\footnotetext{
${ }^{*}$ Correspondence to: John Nolan, Macular Pigment Research Group, Vision Research Centre, Carriganore House, Waterford Institute of Technology, West Campus, Carriganore, Waterford, Ireland. Tel.: +353 51 834074; E-mail: jmnolan@wit.ie.
} 


\section{INTRODUCTION}

Alzheimer's disease (AD) is a chronic and progressive neurodegenerative condition, characterized by a loss of cognitive abilities with impaired executive functioning and change in personality or behavior. Dementia is often and mistakenly used as a synonym for $\mathrm{AD}$, but in fact is an umbrella term describing a variety of conditions that involve the deterioration of cognitive functions, of which $\mathrm{AD}$ is the most common form (accounting for 50-75\% of cases) [1, 2]. Reports show that $\mathrm{AD}$ is the leading cause of dementia, affecting more than 35 million people worldwide [2]. In the United States, a report from 2012 estimated that 5.4 million people currently suffer from $\mathrm{AD}$ [3]. Of note, the incidence of $\mathrm{AD}$ increases exponentially with age [1].

Carotenoids are naturally-occurring plant pigments and important constituents of a healthy diet, because of their established contribution to the antioxidant defense system in the macula and their putative contribution to the antioxidant defense system of the brain $[4,5]$. The carotenoids lutein (L), zeaxanthin (Z) and meso-zeaxanthin (MZ) are found at the macula (the central retina) where they accumulate preferentially relative to the other 42 dietary carotenoids $[6,7]$. This deposit of L, Z, and MZ at the macula is collectively referred to as macular pigment (MP), and MP can be measured in vivo (in units of optical density [OD]), [8] and in vitro (using high performance liquid chromatography [HPLC]) [9].

Research has identified that $\mathrm{L}$ and $\mathrm{Z}$ are also present in the brain (albeit in small amounts, picomolar concentrations), including in the cerebellum, pons, frontal and occipital cortices (MZ analyses were not performed in these studies, because such an analysis requires a separate assay), $[10,11]$ and their presence in these tissues has been confirmed in a recent study [12]. Also, a study in rhesus monkeys reported a positive relationship between retinal and brain concentrations of $\mathrm{L}$ and $\mathrm{Z}$, and significantly so for $\mathrm{L}$ concentrations at the macula with $\mathrm{L}$ concentrations within the cerebellum, occipital cortex, and pons [11].

Furthermore, plasma concentrations of $\mathrm{L}$ and $\mathrm{Z}$ are known to be lower in AD patients when compared with control subjects, with $\mathrm{L}$ concentrations correlating significantly and inversely with dementia severity [13, 14]. Moreover, it has been shown that supplemental $\mathrm{L}$ results in improved performance in a range of tests used to assess cognitive function in unimpaired older women [15]. Renzi et al. have shown that MP levels also relate significantly and positively to performance on a number of measures of cortical function, in healthy elderly subjects [16]. Indeed, a positive relationship between MP and several measures of cognitive function (e.g., Mini-Mental State Examination (MMSE), Montreal cognitive assessment $\left(\mathrm{MoCA}^{\odot}\right)$, prospective memory tests, trail-making tasks, and choice reaction tests) was recently confirmed in a large sample ( $n=4,453$ adults $\geq 50$ years) from the Republic of Ireland [17].

The rationale for the protective, or even cognitionenhancing, effect of the carotenoids in the brain rests primarily on their antioxidant properties [16]. However, other mechanisms whereby the carotenoids in the brain may afford protection, or enhance cognitive function, include: their anti-inflammatory properties; and their role in maintaining the structural integrity of gap junctions; amongst other possible and yetto-be identified mechanisms. Indeed, it is tempting to draw parallels between the putative protective effect of macular carotenoids for AD and age-related macular degeneration (AMD), another age-related disorder affecting the central nervous system (CNS) (the retina being part of the CNS), especially given the antecedents that these two conditions share $[18,19]$. Furthermore, MP's positive relationship with critical fusion frequency (which describes a speed with which signals are processed in the CNS) suggests that MP's constituent carotenoids may play a role in CNS processing [20]. In other words, it is reasonable to hypothesize that MP's constituent carotenoids (L, Z, and $\mathrm{MZ}$ ) may confer protection against $\mathrm{AD}$, and if so, measures of MP may represent an accessible clinical biomarker of concentrations of these potentially important compounds within the brain. However, no study to date has investigated MP levels in patients with AD. The current study, known as the Carotenoids and AgeRelated Dementia Study (CARDS), was designed to investigate whether patients with $\mathrm{AD}$ were comparable to controls (of the same age range) in terms of MP, visual function, and AMD status.

\section{MATERIALS AND METHODS}

\section{Subjects}

This study was conducted in accordance with full sensitivity to the ethical requirements of the patients and control subjects recruited. The study objectives and methodology complied fully with the widelyrecognized international text and codes of practice, such as the Declaration of Helsinki. A protocol was developed specifically for this study by the Principal 
Investigator (JMN) and Consultant Geriatrician (RM) at Waterford Regional Hospital to ensure that informed consent was obtained appropriately, and in keeping with the ethical code germane to obtaining consent from vulnerable subjects (which includes patients with $\mathrm{AD})$.

36 patients with mild to moderate $\mathrm{AD}$ (predominantly moderate) attending the Age-Related Care Unit at Waterford Regional Hospital, Waterford, Ireland were recruited into the study. Mild to moderate $A D$ was defined as an average MMSE score of 14 to 24 with documented difficulties carrying out everyday tasks, and some alteration in behavior. Other screening tests included the clock drawing test and semantic fluency score. Co-morbid diagnoses were documented including vascular risk factors. Current medications were verified including cholinesterase inhibitors and glutamate receptor antagonists. Social and collateral histories were taken for all patients. Non-contrast computed tomography (CT) brain scan was performed to rule out stroke disease.

Thirty-three control subjects were also recruited into the study via newspaper and radio advertisements, and by word of mouth in the local community. Five (15\%) of the subjects recruited into the control group were spouses/partners of the subjects with $\mathrm{AD}$, which was desirable for performing comparison statistics with the $\mathrm{AD}$ group, because of shared environmental variables, but we acknowledge subject recruitment bias represents a weakness inherent in this trial. However, any relevant differences between groups were controlled for during statistical analyses. Ethical approval was granted from the local Waterford South East (of Ireland) Region Ethics Committee prior to the study commencing.

\section{Demographic, medical, ophthalmic, and lifestyle assessment}

A demographic, medical, ophthalmic and lifestyle case history was obtained for each subject at their study visit. Body mass index was calculated $\left(\mathrm{kg} / \mathrm{m}^{2}\right)$ with subject height $(\mathrm{m})$ measured with the Leicester Height Measure, and weight $(\mathrm{kg})$ measured with the SECA weighing scales (SECA, Birmingham, UK). Smoking status was classed as either current smoker (i.e., smoked $\geq 100$ cigarettes in lifetime and at least one cigarette within the last 12 months) or nonsmoker (smoked $\leq 100$ cigarettes in lifetime and none within the last 12 months). Diabetes was assessed by self-report and also by measuring HbA1c in blood (analysis conducted offsite at Biomnis Ireland, Three
Rock Road, Sandyford Business Estate, Dublin 18, Ireland).

\section{Cognitive function assessment}

Cognition was assessed using a selection of validated measures. The MMSE was used to measure the severity of cognitive impairment. This is a rapid, 30 item questionnaire that is commonly used to screen for dementia and track changes over time. A semantic fluency score was obtained using "Animal" as the category (as many exemplars as possible in one minute) and phonemic fluency was measured using the 'FAS Test' (as many words as possible starting with each letter, one minute per letter). Three tasks were chosen from the Cambridge Neuropsychological Test Automated Battery (CANTAB) [21]. All were administered using a finger-operated touch-screen tablet PC using a set of scripted instructions. The Paired Associates Learning task was selected to assess visual memory and learning $[22,23]$. In this task the subject is presented with a set of white boxes, some of which contain a pattern. The objective is to remember the location of each pattern. The test gradually increases in difficulty until the final stage is reached. If a mistake is made then the subject is reminded of the location of the patterns and given another opportunity to respond. The task ends after the final stage is completed or if the subject exceeds a specified number of attempts at any given stage. The scores generated are Total Errors and Total Errors Adjusted. Total Errors is a sum of the errors made across all stages, whereas the adjusted score includes an adjustment made for any stages not reached, allowing it to be comparable to all subjects even if the task was ended prematurely due to cognitive limitation. The Verbal Recognition Memory test assesses immediate and delayed verbal memory and learning under immediate and delayed free-recall and forced-choice recognition conditions [24, 25]. The subject is instructed to immediately recall as many words as possible after being presented with a list of stimuli (on the basis of frequency, word length, and imageability). This process is repeated three times and scores are provided for the number of words correctly recalled in each separate phase, as well as a total correct score. After a short delay, the subjects must then complete a recognition phase with a matched set of distractor stimuli. This stage provides scores based on the total number of correct selections and rejections (of distractor stimuli), as well as false positives. The CANTAB Motor Screening Task was used to assess motor speed and accuracy by instructing the subject to 
touch the center of a series of crosses that are presented on the screen [26].

\section{Best corrected visual acuity}

BCVA was measured with a computerized LogMAR ETDRS test chart (Test Chart 2000 Xpert; Thomson Software Solutions) viewed at 6 meters (m). The Sloan Early Treatment Diabetic Retinopathy Study (ETDRS) letterset was used for this test. At the first incompletely read line, the letters of the line were randomized three times using the testing software's randomization function and an average of three scores was taken. BCVA was recorded as visual acuity rating.

\section{Contrast sensitivity}

CS refers to one's ability to discern an object from its background, and depends on the difference in luminance between the former and the latter, and can be loosely interpreted as 'faintness appreciation'. Letter contrast sensitivity (CS) was assessed using the computerized LogMAR ETDRS test chart (Test Chart 2000 Pro; Thomson Software Solutions) at five different spatial frequencies $(1.2,2.4,6.0,9.6,15.15 \mathrm{cpd})$ [27]. Thirty $(43 \%)$ of the 69 subjects could not perform the high $(15.15 \mathrm{cpd})$ spatial frequency test, and therefore this measure was excluded from analysis. The Sloan optotypes were chosen and subjects were asked to read the letters aloud while fixating on the chart at a distance of $4 \mathrm{~m}$. The letter set was randomized during the test at each change of contrast. The percentage contrast of letter optotypes was decreased in $0.15 \log$ CS steps until the lowest contrast value for which subjects saw at least three letters was reached. The test was then repeated for the other spatial frequencies. Each letter has a nominal $\log$ CS value of 0.03 . Missed letters at any contrast level were noted. The resultant $\log \mathrm{CS}$ value for the subject at a particular spatial frequency was calculated by adding any extra letter(s) and/or subtracting missed letters from best $\log \mathrm{CS}$ value corresponding to the lowest percentage contrast.

\section{Retinal photograph assessment}

45 degree monoscopic color photographs, centered on the macula, were taken in both eyes using a Zeiss Visucam 200 (Carl Zeiss Meditec AG, Jena, Germany). Retinal photographs were assessed for the presence or absence of early AMD, in accordance with the International Classification and Grading System for Age-Related Macular Degeneration by a consultant ophthalmologist with a special interest in retinal disease and with a published track record in grading this condition [28]. In brief, the presence of soft drusen and/or hypo-/hyper-pigmentary changes at the macula were classed as early AMD.

\section{Macular pigment measurement}

The Heidelberg Spectralis ${ }^{\circledR}$ HRA+OCT Multicolor (Heidelberg Engineering GmbH, Heidelberg, Germany) was used to measure MP. The Spectralis utilizes confocal scanning laser ophthalmoscopy imaging with diode lasers and uses dual-wavelength autofluorescence (AF) for measuring MP [29]. Dual-wavelength $\mathrm{AF}$ in this device uses two excitation wavelengths; one that is well-absorbed by MP ( $488 \mathrm{~nm}$, blue) and one that is not well absorbed by the pigment $(518 \mathrm{~nm}$, green). The AF method utilized by the Spectralis has previously been compared with the customized heterochromatic flicker photometry technique for measuring MP, and the measurements recorded from these two devices exhibited excellent concordance [30].

During the measurement, the subject's head is aligned using a head-chin strap and he/she is instructed to fixate on an internal fixation target. A 30-s video is recorded in simultaneous blue $\mathrm{AF}$ and green $\mathrm{AF}$ imaging mode for MP measurement acquisition. The images in the video are then aligned and averaged using the Heidelberg Eye Explorer software (HEYEX, version 1.7.1.0), and a MP density map is created. Central MP at 0.23 degrees eccentricity and MP volume (calculated as MP average times the area under the curve out to 8 degrees eccentricity) are reported here.

\section{Dietary intake of lutein and zeaxanthin}

A subject's weekly intake of carotenoid-rich foods (eggs, broccoli, corn, dark leafy vegetables) was inputted into the L/Z screener to give a carotenoidbased diet score. Values are weighted for frequency of intake of the food and for bioavailability of $\mathrm{L}$ and $\mathrm{Z}$ within these foods. A ranking score reflecting the relative intakes was generated. Evaluation of the L/Z screener against the Willett food frequency questionnaire yielded a positive correlation that was strongly significant $(p<0.01)$. The range of scores on the $\mathrm{L} / \mathrm{Z}$ screener is 0 to 75 . After adding foods with known concentrations of the $\mathrm{L}$ and $\mathrm{Z}$ into the screener, the following estimates can be made: a low dietary carotenoid intake score ranges from $0-15$ ( $\leq 2 \mathrm{mg} /$ day); a medium dietary carotenoid intake 
score ranges from 16-30 (3-13 mg/day); and a high dietary carotenoid intake score ranges from $31-75$ (>13 mg/day).

\section{Serum carotenoid assessment}

\section{Standards and solvents}

The $\mathrm{L}$ and $\mathrm{Z}$ reference standards were purchased from CaroteNature (GmbH Thomas Studer, CEO Gerbestrasse 12 Postfach 1164 CH-3072 Ostermundigen Switzerland). The internal standard (IS) $\alpha$-tocopheryl acetate and all solvents used for extraction and HPLC analysis were supplied by SigmaAldrich (Ireland) and Fisher Scientific (Ireland).

\section{Extraction}

Non-fasting blood samples were collected in $9 \mathrm{ml}$ vacuette tubes containing a ' $Z$ Serum Sep Clot Activator'. The blood samples were allowed to clot at room temperature for approximately $1 \mathrm{~h}$ and then centrifuged at $725 \mathrm{~g}$ for $15 \mathrm{~min}$ in a Gruppe GC 12 centrifuge (Desaga Sarstedt) to separate the serum from the whole blood. The resulting serum samples were stored at $-70^{\circ} \mathrm{C}$ until the time of extraction.

Serum $(0.4 \mathrm{ml})$ was micropipetted into clear $1.5 \mathrm{ml}$ Eppendorf tubes labelled according to subject and visit number. IS $(0.2 \mathrm{ml}), \alpha$-tocopheryl acetate $(250 \mathrm{mg} / \mathrm{l}$ ethanol) and $0.3 \mathrm{ml}$ of butylated hydroxytoluene (BHT) $(250 \mathrm{mg} / \mathrm{l}$ ethanol) were added and extracted into $0.5 \mathrm{ml}$ of heptane using a Vortex Genie-2 (Scientific Industries) at the highest setting for 2 min, followed by centrifugation with a AccuSpin Micro 17 (Fisher Scientific Ireland) for $5 \mathrm{~min}$ at $400 \mathrm{~g}$.

An aliquot of the upper heptane layer $(0.4 \mathrm{ml})$ was removed to a light-resistant Eppendorf tube, and the heptane extraction was repeated once more, adding a further $0.5 \mathrm{ml}$ of heptane to the original residue. The combined extracts were dried under nitrogen and stored at $-70^{\circ} \mathrm{C}$ until HPLC analysis [31].

\section{Analysis}

The chromatographic analysis was performed on an Agilent 1260 Series (Agilent Technologies Limited) equipped with a quaternary pump, autosampler, thermostat column compartment and a photodiode array detector monitoring a wavelength of $450 \mathrm{~nm}$ for serum carotenoids and $292 \mathrm{~nm}$ for the IS. The dried samples were reconstituted in $0.2 \mathrm{ml}$ of $90 \%$ methanol and $10 \%$ methyl-tert-butyl ether (MTBE), vortexed at the lowest setting for $1 \mathrm{~min}$ and pipetted into $2.5 \mathrm{ml}$ vials containing $0.35 \mathrm{ml}$ glass inserts (Sigma-Aldrich). Separations were carried out using a method slightly modified from Yeum et al (see below) [32]. The sample $(0.1 \mathrm{ml})$ was injected via autosampler onto a C30 carotenoid column $(250 \times 4.6 \mathrm{~mm}, 3 \mu \mathrm{m}$ YMC $)$ with a guard column. HPLC mobile phase A consisted of $83 \%$ methanol, $15 \%$ MTBE, and $2 \%$ water with $0.1 \%$ BHT and mobile phase B consisted of $90 \%$ MTBE, $8 \%$ methanol, $2 \%$ water with $0.1 \%$ BHT. At a flow rate of $1 \mathrm{ml} / \mathrm{min}$, the gradient initiated at $5 \%$ solvent $\mathrm{B}$ and increased to $20 \%$ in the first $12 \mathrm{~min}$. Solvent B was increased to $55 \%$ over $8 \mathrm{~min}$ before increasing again to $95 \%$ over the next $7 \mathrm{~min}$. From $27-30 \mathrm{~min}$, solvent B was held at $95 \% \mathrm{~B}$ followed by the system resuming to initial setting at $33 \mathrm{~min}$. The $\mathrm{L}$ and total $\mathrm{Z}$ (co-eluted $\mathrm{Z}$ and MZ) peaks eluted at approximately 10.3 and $12.1 \mathrm{~min}$, respectively. The IS eluted at $8.8 \mathrm{~min}$. Separations were carried out at $16^{\circ} \mathrm{C}$.

\section{Statistics}

The statistical package IBM SPSS version 21 was used for all statistical analyses. The primary outcome measures for this study were MP, measures of vision function variables (i.e., BCVA and CS) and AMD status. Between group differences (AD versus controls) in terms of these outcome measures were first analyzed using independent samples $t$-tests or chi-squared tests, as appropriate. The general linear model, or logistic regression where appropriate, was then used to control for other, possibly confounding, variables, which exhibits significant between group differences (e.g., age, diet, and education). For analyses where the outcome variable was vision-related, we also controlled for MP. The 5\% level of significance was used throughout the analysis, without adjustment for multiple comparisons.

\section{RESULTS}

Table 1 presents the demographic, lifestyle, vision, and cognition data of the $\mathrm{AD}$ and control subjects recruited into the study. The table presents information on outcome variables (i.e., serum L and Z, MP, vision, and AMD status) and on demographic, possibly confounding, variables (e.g., age, body mass index, smoking status). As seen in Table 1, there were statistically significant between group differences in terms of MP, age, diet score, serum L and Z, education, and AMD prevalence, so we controlled for these variables in all subsequent analyses where appropriate. 
Table 1

Demographic, lifestyle, vision, and cognition data of the $\mathrm{AD}$ and control subjects

\begin{tabular}{|c|c|c|c|}
\hline Variables & $\mathrm{AD}(n=36)$ & Control $(n=33)$ & Sig. \\
\hline \multicolumn{4}{|l|}{ Demographic } \\
\hline Age (years) & $80 \pm 7.8$ & $76 \pm 6.6$ & 0.04 \\
\hline $64-68$ & $5(13.9 \%)$ & $2(6.1 \%)$ & 0.28 \\
\hline $69-73$ & $2(5.5 \%)$ & $9(27.2 \%)$ & \\
\hline 74-78 & $8(22.3 \%)$ & $11(33.4 \%)$ & \\
\hline $79-83$ & $8(22.3 \%)$ & $6(18.1 \%)$ & \\
\hline $84-88$ & $7(19.4 \%)$ & $5(15.2 \%)$ & \\
\hline 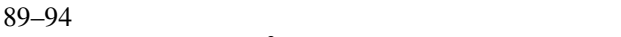 & $6(16.7 \%)$ & $0(0 \%)$ & \\
\hline Body mass index $\left(\mathrm{kg} / \mathrm{m}^{2}\right)$ & $24.9 \pm 5.6$ & $26.2 \pm 3.4$ & \\
\hline Exercise (total exercise per week) & $179 \pm 214.5$ & $221 \pm 160.0$ & 0.38 \\
\hline Diet (estimated lutein and zeaxanthin intake) & $15 \pm 8.4$ & $23 \pm 14.0$ & 0.01 \\
\hline Serum lutein $(\mu \mathrm{mol} / \mathrm{l})$ & $0.217 \pm 0.112$ & $0.295 \pm 0.175$ & 0.036 \\
\hline Serum zeaxanthin $(\mu \mathrm{mol} / \mathrm{l})$ & $0.049 \pm 0.034$ & $0.074 \pm 0.041$ & 0.010 \\
\hline Education (total years in education) & $10 \pm 4.3$ & $14 \pm 4.3$ & 0.001 \\
\hline Smoking (\% current) & $8.60 \%$ & $9.70 \%$ & 0.88 \\
\hline Gender ( $\%$ female $)$ & $64 \%$ & $48 \%$ & 0.2 \\
\hline Diabetes (\% with diabetes) & 9.7 & 6.5 & 0.259 \\
\hline \multicolumn{4}{|l|}{ Vision } \\
\hline MP 0.23 & $0.40 \pm 0.20$ & $0.58 \pm 0.17$ & $<0.001$ \\
\hline MP vol & $3700 \pm 2833$ & $6776 \pm 2834$ & $<0.001$ \\
\hline BCVA & $88.4 \pm 11.4$ & $95.8 \pm 8.4$ & 0.004 \\
\hline CS1.2 (cpd) & $1.49 \pm 0.22$ & $1.77 \pm .23$ & $<0.001$ \\
\hline $\mathrm{CS} 2.4$ (cpd) & $1.47 \pm 0.29$ & $1.76 \pm 0.26$ & $<0.001$ \\
\hline CS6.0 (cpd) & $1.18 \pm 0.31$ & $1.43 \pm 0.24$ & 0.001 \\
\hline CS9.6 (cpd) & $0.91 \pm 0.34$ & $1.19 \pm 0.27$ & 0.001 \\
\hline AMD (\% with AMD) & $44.40 \%$ & $18.20 \%$ & 0.019 \\
\hline \multicolumn{4}{|l|}{ Retinal thickness } \\
\hline Central min thickness & $231 \pm 36$ & $224 \pm 27$ & 0.365 \\
\hline Central max thickness & $321 \pm 34$ & $320 \pm 23$ & 0.917 \\
\hline Central mean thickness & $277 \pm 32$ & $275 \pm 26$ & 0.800 \\
\hline Average thickness & $324 \pm 29$ & $333 \pm 24$ & 0.182 \\
\hline \multicolumn{4}{|l|}{ Cognition } \\
\hline MMSE & $18.8 \pm 3.7$ & $29.0 \pm 1.7$ & $<0.001$ \\
\hline Semantic fluency score & $6.1 \pm 3.2$ & $15.4 \pm 5.1$ & $<0.001$ \\
\hline Phonemic fluency score & $15.7 \pm 10.0$ & $33.5 \pm 13.8$ & $<0.001$ \\
\hline MOT (mean latency) & $1860 \pm 799$ & $1322 \pm 571$ & 0.005 \\
\hline MOT (mean error) & $11.9 \pm 3.14$ & $9.5 \pm 2.8$ & 0.003 \\
\hline VRM (phase 1) & $1.4 \pm 1.2$ & $5.2 \pm 2.7$ & $<0.001$ \\
\hline VRM (phase 2) & $2.5 \pm 1.6$ & $7.4 \pm 2.7$ & $<0.001$ \\
\hline VRM (phase 3) & $3.3 \pm 2.1$ & $8.2 \pm 2.9$ & $<0.001$ \\
\hline VRM (correct words) & $1.40 \pm 1.17$ & $5.24 \pm 2.69$ & $<0.001$ \\
\hline VRM delayed stage (correct words) & $1.3 \pm 3.73$ & $7.2 \pm 4.6$ & $<0.001$ \\
\hline VRM delayed stage (recognition total correct) & $17 \pm 3.3$ & $23 \pm 1.4$ & $<0.001$ \\
\hline VRM delayed stage (recognition total false positive) & $4.6 \pm 3.5$ & $0.68 \pm 0.91$ & $<0.001$ \\
\hline PAL (total errors) & $135.5 \pm 14.3$ & $68 \pm 39$ & $<0.001$ \\
\hline PAL (total errors adjusted) & $24.6 \pm 11.4$ & $17.1 \pm 11.02$ & 0.01 \\
\hline
\end{tabular}

Data displayed are mean \pm standard deviation for interval data and percentages for categorical data. Variables, variables analyzed in the study; $\mathrm{AD}$, subjects recruited into the study confirmed as having mild to moderate Alzheimer's disease; Control, subjects free of mild to moderate $\mathrm{AD}$ and of similar age to the AD subjects; Sig., the statistical difference (p value) between AD and control subjects assessed using independent samples $t$-tests or chi-squared depending on the variable of interest; Exercise, total exercise for any sporting activity measured as minutes per week; Diet, estimate of dietary intake of L and Z; Smoking, current (smoked $\geq 100$ cigarettes in lifetime and at least one cigarette within the last 12 months) or non-smoking (smoked $\leq 100$ cigarettes in lifetime and none within the last 12 months); Diabetes, $\%$ of subjects with diabetes as confirmed by self-report and by HbA1c analysis; MP 0.23 , central macular pigment measured at 0.23 degrees eccentricity measured using the Heidelberg Spectralis ${ }^{\circledR}$. MP vol, a volume of MP calculated as MP average times the area under the curve out to 8 degrees eccentricity (measured using the Heidelberg Spectralis ${ }^{\circledR}$ ). BCVA, best corrected visual acuity. CS 1.2, CS 2.4, CS 6.0 and CS 9.6, letter contrast sensitivity measured using the Thomson Software Solutions at 1.2, 2.4, 6.0, and 9.6 cycles per degree. AMD, age-related macular degeneration; Central min thickness, the minimum thickness within a $1 \mathrm{~mm}$ diameter of the fovea. Central max thickness, the maximum thickness within a $1 \mathrm{~mm}$ diameter of the fovea; Central mean thickness, average thickness within a $1 \mathrm{~mm}$ diameter of the fovea; Average thickness, average thickness at $2 \mathrm{~mm}$ nasally; MMSE, Mini Mental State Examination; Semantic fluency score, a semantic fluency (categorical verbal fluency) score obtained from the number of animals named by the subject in 1 minute; Phonemic fluency score, A phonemic fluency (word fluency) score generated by 


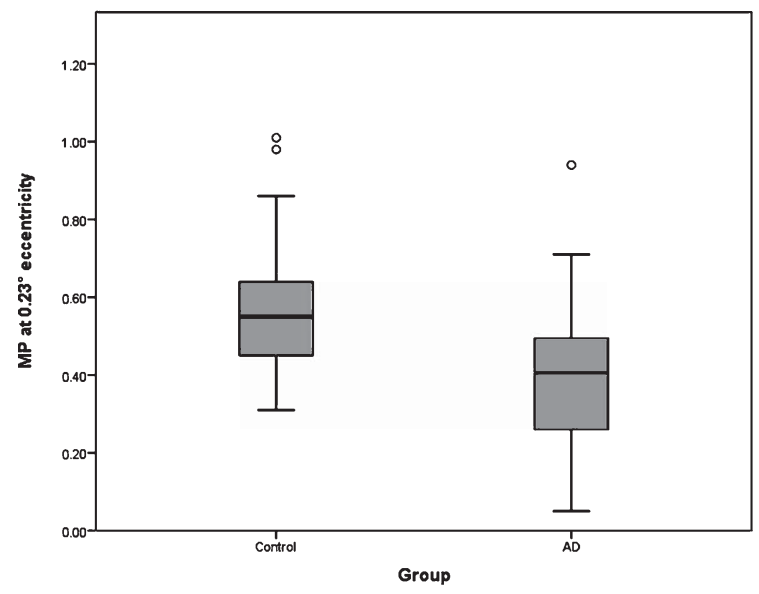

Fig. 1. Boxplots of macular pigment optical density at $0.23^{\circ}$ for the Alzheimer's disease (AD) and control groups.

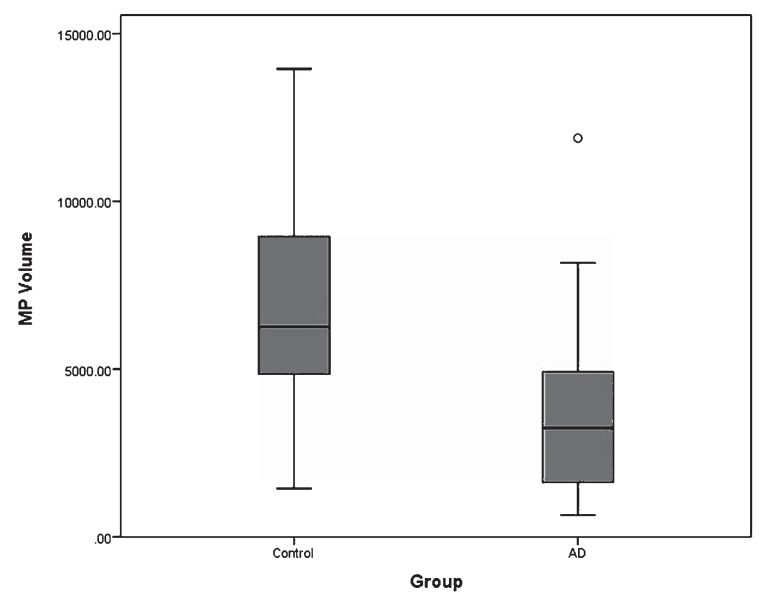

Fig. 2. Boxplots of macular pigment optical density volume for the Alzheimer's disease (AD) and control groups.

\section{Serum L and $Z$ concentrations}

Serum concentrations of $\mathrm{L}$ and $\mathrm{Z}$ in the $\mathrm{AD}$ group were significantly lower than serum concentrations of these carotenoids in the control group $(p=0.036$ and $p=0.01$, respectively). The statistical significance of this relationship persisted after controlling for age and education ( $p=0.05$ and $p=0.014$, for $\mathrm{L}$ and $\mathrm{Z}$, respectively). The significance disappears, however, if we also control for diet in these analyses, and this is likely due to "confounding", as diet is significantly related to the dependent variable ( $\mathrm{L}$ or $\mathrm{Z}$ ) and to $\mathrm{AD}$ status.

\section{Macular pigment}

Central MP (i.e., at $0.23^{\circ}$ ) and MP volume were both significantly lower in the AD group when compared to the control group (see Table 1 and Figs. 1 and 2; $p<0.001$ for both). Of note, for MP at $0.23^{\circ}$, the statistical significance of this relationship persisted ( $p=0.002)$ after controlling for age, diet, education, and presence of AMD. This was also true for MP volume $(p=0.001)$.

\section{Visual function}

\section{Best corrected visual acuity}

BCVA was significantly lower in the AD group when compared to the control group (see Table 1 and Fig. 3; $p=0.004)$. The statistical significance of this relationship persisted $(p=0.028)$ after controlling for age, but was attenuated to borderline significance $(p=0.077)$ after controlling for MP, age, diet, education, and presence of AMD.

\section{Contrast sensitivity}

CS (at all spatial frequencies measured) was significantly lower in the AD group when compared to the control group (see Table 1 and Fig. 4). The statistical significance of this relationship persisted after controlling for MP, age, diet, education, and presence of AMD (significance ranging from $p<0.001$ to 0.018 ).

\section{$A M D$}

Prevalence of AMD was significantly higher in the AD group when compared to the control group (Table 1, $p=0.019$ ). However, after controlling for age, education, MP, and diet, this observed between-group effect is no longer significant $(p=0.147)$; as with the analysis of $\mathrm{L}$ and $\mathrm{Z}$ reported above, there is a likely con-

the total number of words produced for the each of the letters F, A, and S, in 1 minute; MOT (mean latency), motor screening task measures the subject's speed of response; MOT (mean error), motor screening task measures the accuracy of the subject's pointing at cross targets; VRM (phase 1), VRM (phase 2), VRM (phase 3), VRM (correct words), VRM delayed stage (correct words), VRM delayed stage (recognition total correct), VRM delayed stage (recognition total false positive), Verbal Recognition Memory tests which assess immediate and delayed memory of verbal information under free recall and forced choice recognition conditions; PAL (total errors) and PAL (total errors adjusted), Paired Associates Learning tests which measure visual memory and new learning of the subjects; PAL (total errors), a sum of the errors made across all stages; PAL (total errors adjusted), the adjusted score and includes an adjustment made for any stages not reached, allowing it to be comparable to all subjects even if the task was ended prematurely due to cognitive limitation. 


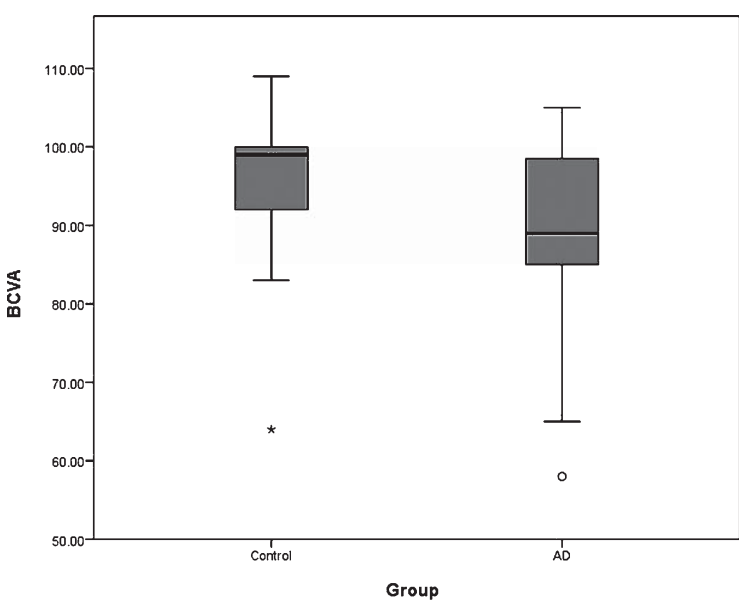

Fig. 3. Boxplots of best corrected visual acuity for the Alzheimer's disease $(\mathrm{AD})$ and control groups.

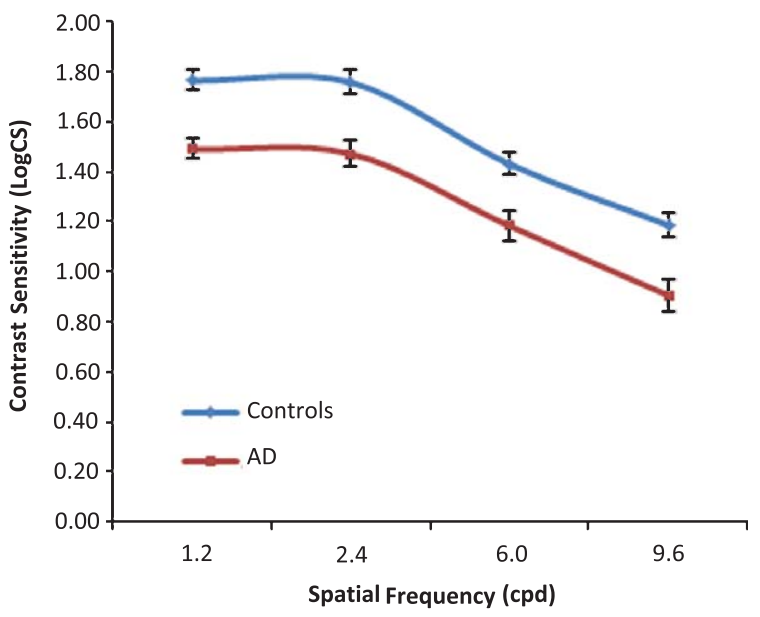

Fig. 4. Contrast sensitivity curves for the Alzheimer's disease (AD) and control groups.

founding effect here. When controlling just for age, however, the group effect exhibits borderline significance $(p=0.069)$.

\section{DISCUSSION}

Our study has demonstrated that patients with mild to moderate $\mathrm{AD}$ compared to a control group have 1) a relative lack of MP; 2) significantly poorer visual function; and 3) higher occurrence of AMD. For the most part, these findings persist, even after controlling for confounding variables. We discuss below the implications of these findings, and propose explanations, where possible, for the observed associations.
Firstly, with regard to our finding of a higher occurrence of $\mathrm{AMD}$ in the $\mathrm{AD}$ group, we know that $\mathrm{AMD}$ and $\mathrm{AD}$ are neurodegenerative disorders that share several antecedents, which can be classed as sociodemographic, cardiovascular and genetic [19, 33, 34]. For certain, these degenerative disorders are clustered towards the end of life and are thought to be the result of cumulative and lifelong oxidative injury (consistent with the free radical theory of ageng) [35]. Furthermore, $\mathrm{AMD}$ and $\mathrm{AD}$ are characterized by the formation of drusen in the retina and senile plaques in the CNS, respectively [18]. The pathologic changes observed in these conditions are the result of oxidation [36, 37] and inflammation $[38,39]$ within their respective tissues, and their molecular compositions are comparable. With regard to risk factors for $\mathrm{AMD}$ and $\mathrm{AD}$, age is the principal common risk factor, but female sex and poor socio-economic background are also important $[33,34]$. Also, with regard to cardiovascular risk factors common to $\mathrm{AMD}$ and $\mathrm{AD}$, hypertension, tobacco use, obesity, and dyslipidemia are each associated with increased risk for developing these conditions $[40,41]$.

With regard to the genetic risk factors, $\mathrm{AD}$ and $\mathrm{AMD}$ also share associations with several genetic polymorphisms that influence molecular pathways important in the pathogenesis of each of these conditions, including the complement system, lipid homeostasis, and vascular endothelial function [42-44]. Of note, our finding of a higher occurrence of AMD in patients with $\mathrm{AD}$ is consistent with previous reports which have shown that patients with AMD have an elevated risk for AD [45], and also consistent with observations that cognitive function is reduced amongst sufferers of AMD [46].

In summary, the commonalities between $\mathrm{AD}$ and AMD, including genetic and environmental risk factors, help explain, at least in part, our finding of a higher occurrence of AMD in the AD group when compared to controls, and perhaps suggest a role for a shared perspective for exploring means of preventing or retarding the onset or progression of these disorders. Indeed, there is consistency across studies investigating the protective role of antioxidants, which include the macular carotenoids, for retinal health and AMD, which had demonstrated a reduced progression to advanced AMD following supplemental antioxidants $[47,48]$. Furthermore, there is good reason to believe that augmentation of MP may play a role in the prevention of AMD [41]. Indeed, the rationale behind this notion is biologically plausible and there is an emerging and evidence-based consensus that supplementation with all three macular carotenoids 
is likely to be the most promising strategy designed to delay or prevent the onset or progression of AMD $[49,50]$.

However, questions remain with respect to advice given by eye care professionals regarding antioxidant supplementation in patients with AMD or at risk of developing this condition, and a lack of clarity persists around several issues including a daily recommended intake of each of the macular carotenoids and whether the observed benefits in AREDS2 will pertain outside of the context of a clinical trial [51].

It is known that plasma concentrations of $\mathrm{L}$ and $\mathrm{Z}$ are lower in AD patients when compared with control subjects, with L concentrations correlating significantly and inversely with dementia severity [13, 14]. Moreover, a recent publication using the Third National Health and Nutrition Examination Survey (NHANES III) database and the NHANES III Linked Mortality File found that high serum concentrations of lycopene, $\mathrm{L}$ and $\mathrm{Z}$ (but not other carotenoids) were associated with a significantly lower risk of mortality attributable to $\mathrm{AD}$ [52]. These findings have been confirmed in our current study in that the $\mathrm{AD}$ subjects had significantly less (on average) serum concentrations of $\mathrm{L}$ and $\mathrm{Z}$ when compared to the control group. It is important to point out that (as expected) dietary intake of $\mathrm{L}$ and $\mathrm{Z}$ (which was also significantly less in the AD subjects) correlated well with serum concentrations of $\mathrm{L}$ and $\mathrm{Z}$ in our study $(r=0.572, p<0.001$ and $r=0.445, p<0.001$ ), suggesting that the lower circulating serum concentrations of these carotenoids in $\mathrm{AD}$ patients when compared to controls is due (at least in part) to poor diets in patients with AD.

With the above in mind, our finding that patients with AD have significantly less MP when compared to controls (even after controlling for dietary differences) is all the more provocative, and may reflect a parallel and relative lack of MP's constituent carotenoids in brain tissue. Of interest, it is known that MP correlates with concentrations of the macular carotenoids in primate brain tissue, [11] and it is therefore tempting to hypothesize that measures of MP (which can be uniquely performed in vivo $[53,54]$ ) should be explored as a possible biomarker for concentrations of these compounds in brain tissue and/or cognitive function in health and/or disease.

Carotenoids in the human brain were first identified by Mathews-Roth et al. in 1976 in two patients receiving $\beta$-carotene supplements [55]. Later, the first report of individually measured carotenoids in the human brain was published by Craft et al., who reported that between $66 \%$ and $77 \%$ of the carotenoids (including substantial quantities of $\mathrm{L}$ and Z) in the human brain were present in the occipital and frontal cortices [10]. Of interest, a recent study by Johnson et al. confirmed the presence of $\mathrm{L}$ and $\mathrm{Z}$ in several parts of the human brain including frontal cortex, occipital cortex, temporal cortex, and in large amounts in the cerebellum [12].

In the report by Johnson et al., serum $\mathrm{L}$ and $\mathrm{Z}$ concentrations were most consistently related to better cognitive performance in the elderly population studied (>80 years), including Global Deterioration Scale, Controlled Oral Word Association Test, Wechsler Adult Intelligence Scale-III Similarities Subtest and the Behavioral Dyscontrol Scale [12]. Consistent with the above findings (and suggestive of a beneficial) role for carotenoids with respect to cognitive function), a positive relationship between MP and several measures of cognitive function (e.g., MMSE, MoCA, Prospective Memory Tests, Trail-Making Tasks, and Choice Reaction Tests) was recently confirmed in a large sample ( $n=4,453$ adults $\geq 50$ years) from the Republic of Ireland [17]. Also, a recent study from the US has reported positive and significant relationships, in older people, between MP and global cognition, verbal learning and fluency, recall, processing speed, perceptual speed [56].

Finally, our finding that AD subjects have significantly poorer vision when compared to controls needs to be emphasized. In brief, the AD subjects had significantly poorer visual function, as reflected in measures of BCVA and CS, than control subjects, and the observed differences are clinically meaningful. We investigated if the poor vision observed in AD patients when compared to controls was due to the higher prevalence of AMD seen in these patients, and we found that AMD was not a significant predictor of visual function (likely due the fact that the AMD observed in these patients was early stage, and therefore not visually consequential). In other words, our data suggests that the poor vision observed in $\mathrm{AD}$ patients was not due to their high prevalence of AMD, as AD patients without AMD also had significantly poorer vision when compared to the control group. This is a novel finding and supports the view that appropriate strategies should be put in place to try and protect and enhance visual function in these vulnerable patients. Of importance, it has already been shown that enrichment of MP with macular carotenoid supplementation enhances visual function in subjects with and without retinal disease [49, 57-60].

In conclusion, there is an established evidence base that MP, made up of its constituent carotenoids L, Z 
and MZ, protects against AMD progression, [61] and there is a growing body of evidence that MP also plays a role in prevention of this condition. The presence of $\mathrm{L}$ and $\mathrm{Z}$ in brain tissue has also been confirmed, [10] and brain carotenoid concentrations are positively related to MP [11]. There is a sound and biologically plausible rationale whereby $\mathrm{L}, \mathrm{Z}$ and $\mathrm{MZ}$ may be important in the prevention and/or delay in the onset of $\mathrm{AD}$. Our main finding that AD patients have significantly less $\mathrm{MP}$ and poorer vision when compared to controls merits further investigation, and a clinical trial designed to investigate the impact of macular carotenoid supplementation with respect to MP, visual function and cognitive function among AD sufferers is merited. Furthermore, the possibility that measures of MP may have a role as a non-invasive biomarker of cognitive function and/or cognitive impairment, or risk thereof, warrants further study.

\section{ACKNOWLEDGMENTS}

We would like to thank the Howard Foundation, Cambridge, CB22 5LA, United Kingdom for supporting this research. We would like to acknowledge Dr. Robert Coen from the Mercer's Institute for Successful Ageing, St. James's Hospital, Dublin, Ireland for his assistance and advice with the measurements of cognitive function. We would like to acknowledge Cambridge Cognition, UK for guidance with respect to the assessment of cognitive function. Also, we would like to thank all the staff at the Waterford Regional Hospital, Age-Related Care Unit and at the Vision Research Centre, Waterford Institute of Technology for assisting this study.

Authors' disclosures available online (http://www.jalz.com/disclosures/view.php?id=2318).

\section{REFERENCES}

[1] Povova J, Ambroz P, Bar M, Pavukova V, Sery O, Tomaskova $\mathrm{H}$, Janout V (2012) Epidemiological of and risk factors for Alzheimer's disease: A review. Biomed Pap Med Fac Univ Palacky Olomouc Czech Repub 156, 108-114.

[2] Querfurth HW, LaFerla FM (2010) Alzheimer's disease. $N$ Engl J Med 362, 329-344.

[3] (2012) 2012 Alzheimer's disease facts and figures. Alzheimers Dement 8, 131-168.

[4] Khachik F, Bernstein PS, Garland DL (1997) Identification of lutein and zeaxanthin oxidation products in human and monkey retinas. Invest Ophthalmol Vis Sci 38, 1802-1811.

[5] Johnson EJ (2012) A possible role for lutein and zeaxanthin in cognitive function in the elderly. Am J Clin Nutr 96, 1161S$1165 \mathrm{~S}$.

[6] Khachik F, Beecher GR, Goli MB (1991) Separation, identification and quantification of carotenoids in fruits, vegetables and human plasma by high performance liquid chromatography. Pure Appl Chem 63, 71-80.

[7] Bernstein PS, Khachik F, Carvalho LS, Muir GJ, Zhao DY, Katz NB (2001) Identification and quantitation of carotenoids and their metabolites in the tissues of the human eye. Exp Eye Res 72, 215-223.

[8] Loane E, Stack J, Beatty S, Nolan JM (2007) Measurement of macular pigment optical density using two different heterochromatic flicker photometers. Curr Eye Res 32, 555-564.

[9] Bone RA, Landrum JT, Fernandez L, Tarsis SL (1988) Analysis of the macular pigment by HPLC - Retinal distribution and age study. Invest Ophthalmol Vis Sci 29, 843-849.

[10] Craft NE, Haitema TB, Garnett KM, Fitch KA, Dorey CK (2004) Carotenoid, tocopherol, and retinol concentrations in elderly human brain. J Nutr Health Aging 8, 156-162.

[11] Vishwanathan R, Neuringer M, Snodderly DM, Schalch W, Johnson EJ (2013) Macular lutein and zeaxanthin are related to brain lutein and zeaxanthin in primates. Nutr Neurosci 16, 21-29.

[12] Johnson EJ, Vishwanathan R, Johnson MA, Hausman DB, Davey A, Scott TM, Green RC, Miller LS, Gearing M, Woodard J, Nelson PT, Chung HY, Schalch W, Wittwer J, Poon LW (2013) Relationship between serum and brain carotenoids, alpha-tocopherol, and retinol concentrations and cognitive performance in the oldest old from the Georgia Centenarian Study. J Aging Res 2013, 951786.

[13] Rinaldi P, Polidori MC, Metastasio A, Mariani E, Mattioli P, Cherubini A, Catani M, Cecchetti R, Senin U, Mecocci P (2003) Plasma antioxidants are similarly depleted in mild cognitive impairment and in Alzheimer's disease. Neurobiol Aging 24, 915-919.

[14] Wang W, Shinto L, Connor WE, Quinn JF (2008) Nutritional biomarkers in Alzheimer's disease: The association between carotenoids, n-3 fatty acids, and dementia severity. $J$ Alzheimers Dis 13, 31-38.

[15] Johnson EJ, McDonald K, Caldarella SM, Chung HY, Troen AM, Snodderly DM (2008) Cognitive findings of an exploratory trial of docosahexaenoic acid and lutein supplementation in older women. Nutr Neurosci 11, 75-83.

[16] Renzi LM, Iannaccone A, Johnson E, Kritchevsky S (2008) The relation between serum xanthophylls, fatty acids, macular pigment and cognitive function in the Health ABC Study. FASEB J 22, 877.5.

[17] Feeney J, Finucane C, Savva GM, Cronin H, Beatty S, Nolan J, Kenny RA (2013) Low macular pigment optical density is associated with lower cognitive performance in a large population-based sample of older adults. Neurobiol Aging 34, 2449-2456.

[18] Ohno-Matsui K (2011) Parallel findings in age-related macular degeneration and Alzheimer's disease. Prog Retin Eye Res 30, 217-238.

[19] Kaarniranta K, Salminen A, Haapasalo A, Soininen H, Hiltunen M (2011) Age-related macular degeneration (AMD): Alzheimer's disease in the eye? J Alzheimers Dis 24, 615-631.

[20] Hammond BR Jr, Wooten BR (2005) CFF thresholds: Relation to macular pigment optical density. Ophthalmic Physiol Opt 25, 315-319.

[21] Robbins TW, James M, Owen AM, Sahakian BJ, McInnes L, Rabbitt P (1994) Cambridge Neuropsychological Test Automated Battery (CANTAB): A factor analytic study of a large sample of normal elderly volunteers. Dementia 5, 266-281.

[22] Rusted JM, Warburton DM (1988) The effects of scopolamine on working memory in healthy young volunteers. Psychopharmacology (Berl) 96, 145-152. 
[23] Sahakian BJ, Morris RG, Evenden JL, Heald A, Levy R, Philpot M, Robbins TW (1988) A comparative study of visuospatial memory and learning in Alzheimer-type dementia and Parkinson's disease. Brain 111(Pt 3), 695-718.

[24] Need AC, Attix DK, McEvoy JM, Cirulli ET, Linney KL, Hunt P, Ge D, Heinzen EL, Maia JM, Shianna KV, Weale ME, Cherkas LF, Clement G, Spector TD, Gibson G, Goldstein DB (2009) A genome-wide study of common SNPs and CNVs in cognitive performance in the CANTAB. Hum Mol Genet 18, 4650-4661.

[25] Yurko-Mauro K, McCarthy D, Rom D, Nelson EB, Ryan AS, Blackwell A, Salem N Jr, Stedman M(2010) Beneficial effects of docosahexaenoic acid on cognition in age-related cognitive decline. Alzheimers Dement 6, 456-464.

[26] Owen AM, Downes JJ, Sahakian BJ, Polkey CE, Robbins TW (1990) Planning and spatial working memory following frontal lobe lesions in man. Neuropsychologia $\mathbf{2 8}$, 1021-1034.

[27] Charalampidou S, Nolan J, Loughman J, Stack J, Higgins G, Cassidy L, Beatty S (2011) Psychophysical impact and optical and morphological characteristics of symptomatic non-advanced cataract. Eye (Lond) 25, 1147-1154.

[28] Bird AC, Bressler NM, Bressler SB, Chisholm IH, Coscas G Davis DM, de Jong PT, Klaver CC, Klein BE, Klein R, et al. (1995) An international classification and grading system for age-related maculopathy and age-related macular degeneration. The International ARM Epidemiological Study Group. Surv Ophthalmol 39, 367-374.

[29] Delori FC (2004) Autofluorescence method to measure macular pigment optical densities fluorometry and autofluorescence imaging. Arch Biochem Biophys 430, 156-162.

[30] Dennison JL, Stack J, Beatty S, Nolan JM (2013) Concordance of macular pigment measurements obtained using customized heterochromatic flicker photometry, dual-wavelength autofluorescence, and single-wavelength reflectance. Exp Eye Res 116C, 190-198.

[31] Meagher KA, Thurnham DI, Beatty S, Howard AN, Connolly E, Cummins W, Nolan JM (2012) Serum response to supplemental macular carotenoids in subjects with and without age-related macular degeneration. Br J Nutr 110, 289-300.

[32] Yeum KJ, Booth SL, Sadowski JA, Liu C, Tang G, Krinsky NI, Russell RM (1996) Human plasma carotenoid response to the ingestion of controlled diets high in fruits and vegetables. Am J Clin Nutr 64, 594-602.

[33] Blennow K, de Leon MJ, Zetterberg H (2006) Alzheimer's disease. Lancet 368, 387-403.

[34] Lim LS, Mitchell P, Seddon JM, Holz FG, Wong TY (2012) Age-related macular degeneration. Lancet 379, 1728-1738.

[35] Harman D (1992) Free radical theory of aging. Mutat Res 275, 257-266

[36] Beatty S, Koh HH, Henson D, Boulton M (2000) The role of oxidative stress in the pathogenesis of age-related macular degeneration. Surv Ophthalmol 45, 115-134.

[37] Bonda DJ, Wang X, Perry G, Nunomura A, Tabaton M, Zhu X, Smith MA (2010) Oxidative stress in Alzheimer disease: A possibility for prevention. Neuropharmacology $\mathbf{5 9}$, 290-294.

[38] Anderson DH, Mullins RF, Hageman GS, Johnson LV (2002) A role for local inflammation in the formation of drusen in the aging eye. Am J Ophthalmol 134, 411-431.

[39] Walsh S, Aisen PS (2004) Inflammatory processes and Alzheimer's disease. Expert Rev Neurother 4, 793-798.

[40] Qiu C (2012) Preventing Alzheimer's disease by targeting vascular risk factors: Hope and gap. J Alzheimers Dis 32, 721-731.
[41] Nolan JM, Stack J, O' DO, Loane E, Beatty S (2007) Risk factors for age-related maculopathy are associated with a relative lack of macular pigment. Exp Eye Res 84, 61-74.

[42] Vagnucci AH Jr, Li WW (2003) Alzheimer's disease and angiogenesis. Lancet 361, 605-608.

[43] Proitsi P, Lupton MK, Dudbridge F, Tsolaki M, Hamilton G, Daniilidou M, Pritchard M, Lord K, Martin BM, Johnson J, Craig D, Todd S, McGuinness B, Hollingworth P, Harold D, Kloszewska I, Soininen H, Mecocci P, Velas B, Gill M, Lawlor B, Rubinsztein DC, Brayne C, Passmore PA, Williams J, Lovestone S, Powell JF (2012) Alzheimer's disease and agerelated macular degeneration have different genetic models for complement gene variation. Neurobiol Aging 33, 1843-17.

[44] Zetterberg M, Landgren S, Andersson ME, Palmer MS, Gustafson DR, Skoog I, Minthon L, Thelle DS, Wallin A, Bogdanovic N, Andreasen N, Blennow K, Zetterberg H (2008) Association of complement factor $\mathrm{H} \mathrm{Y} 402 \mathrm{H}$ gene polymorphism with Alzheimer's disease. Am J Med Genet B Neuropsychiatr Genet 147B, 720-726.

[45] Klaver CC, Ott A, Hofman A, Assink JJ, Breteler MM, de Jong PT (1999) Is age-related maculopathy associated with Alzheimer's Disease? The Rotterdam Study. Am J Epidemiol 150, 963-968.

[46] Clemons TE, Rankin MW, McBee WL (2006) Cognitive impairment in the Age-Related Eye Disease Study: AREDS report no. 16. Arch Ophthalmol 124, 537-543.

[47] Chew E, The AREDS2 Study Group (2013) Lutein + zeaxanthin and omega-3 fatty acids for age-related macular degeneration: The Age-Related Eye Disease Study 2 (AREDS2) randomized clinical trial. JAMA 309, 2005-2015.

[48] Beatty S, Chakravarthy U, Nolan JM, Muldrew KA, Woodside JV, Denny F, Stevenson MR (2013) Secondary outcomes in a clinical trial of carotenoids with coantioxidants versus placebo in early age-related macular degeneration. Ophthalmology 120, 600-606.

[49] Loughman J, Nolan JM, Howard AN, Connolly E, Meagher K, Beatty S (2012) The impact of macular pigment augmentation on visual performance using different carotenoid formulations. Invest Ophthalmol Vis Sci 53, 7871-7880.

[50] Nolan JM, Akkali MC, Loughman J, Howard AN, Beatty S (2012) Macular carotenoid supplementation in subjects with atypical spatial profiles of macular pigment. Exp Eye Res 101, 9-15.

[51] Musch DC (2014) Evidence for including lutein and zeaxanthin in oral supplements for age-related macular degeneration. JAMA Ophthalmol 132, 139-141.

[52] Min JY, Min KB (2013) Serum lycopene, lutein and zeaxanthin, and the risk of Alzheimer's disease mortality in older adults. Dement Geriatr Cogn Disord 37, 246-256.

[53] Wooten BR, Hammond BR, Land RI, Snodderly DM (1999) A practical method for measuring macular pigment optical density. Invest Ophthalmol Vis Sci 40, 2481-2489.

[54] Delori FC, Goger DG, Hammond BR, Snodderly DM, Burns SA (2001) Macular pigment density measured by autofluorescence spectrometry: Comparison with reflectometry and heterochromatic flicker photometry. J Opt Soc Am A Opt Image Sci Vis 18, 1212-1230.

[55] Mathews-Roth MM, Abraham AA, Gabuzda TG (1976) Betacarotene content of certain organs from two patients receiving high doses of beta-carotene. Clin Chem 22, 922-924.

[56] Vishwanathan R, Iannaccone A, Scott TM, Kritchevsky SB, Jennings BJ, Carboni G, Forma G, Satterfield S, Harris T, Johnson KC, Schalch W, Renzi LM, Rosano C, Johnson EJ (2014) Macular pigment optical density is related to cognitive function in older people. Age Ageing 43, 271-275. 
[57] Scott IU, Feuer WJ, Jacko JA (2002) Impact of visual function on computer task accuracy and reaction time in a cohort of patients with age-related macular degeneration. Am J Ophthalmol 133, 350-357.

[58] Stringham JM, Garcia PV, Smith PA, McLin LN, Foutch BK (2011) Macular pigment and visual performance in glare: Benefits for photostress recovery, disability glare, and visual discomfort. Invest Ophthalmol Vis Sci 52, 7406-7415.

[59] Bartlett HE, Eperjesi F (2008) A randomised controlled trial investigating the effect of lutein and antioxidant dietary supplementation on visual function in healthy eyes. Clin Nutr 27, 218-227.
[60] Sasamoto Y, Gomi F, Sawa M, Tsujikawa M, Nishida K (2011) Effect of 1-year lutein supplementation on macular pigment optical density and visual function. Graefes Arch Clin Exp Ophthalmol 249, 1847-1854.

[61] Sabour-Pickett S, Nolan JM, Loughman J, Beatty S (2011) A review of the evidence germane to the putative protective role of the macular carotenoids for age-related macular degeneration. Mol Nutr Food Res 56, 270-286. 